\title{
La precipitación en el Valle del Mantaro y las anomalías de temperatura del Océano Atlántico Tropical
}

\author{
Mantaro Valley rainfall and the tropical Atlantic Ocean Temperature anomalies
}

\author{
Juan Carlos Chang ${ }^{1}$
}

\section{Resumen}

Se ha buscado una relación entre las anomalías de temperatura de la superficie del mar en el Atlántico tropical y la precipitación en la estación húmeda (octubre-marzo) del valle del Mantaro. La variabilidad de las anomalías de temperatura superficial del océano Atlántico tropical explica el 64,7 \% de la variancia de la precipitación en los meses húmedos (octubre a marzo) en el valle del Mantaro. La variancia explicada fue calculada usando regresión múltiple lineal y validación cruzada (Montgomery, 2001). Los resultados sugieren que las anomalías correlacionadas están vinculadas al movimiento de la zona de convergencia intertropical. Se ha usado una serie de 26 años de precipitación en la estación de Huayao, provincia de Chupaca. Las anomalías de la temperatura superficial del mar fueron estimadas usando los índices TNA y TSA (Tropical North Atlantic y Tropical South Atlantic), descritas por el Laboratorio de Investigación de Ciencias de la Tierra (ESRL) de la National Oceanic \& Atmospheric Administration (NOAA).

Palabras clave: precipitación Valle Mantaro; índices oceánicos; TNA índice del Atlántico Tropical Norte; TSA índice del Atlántico Tropical Sur.

\begin{abstract}
A relationship between sea surface temperature anomalies in the tropical Atlantic and the wet season (October-March) total rainfall at Mantaro Valley was searched. The sea surface temperature anomaly variability explained 64,7 \% of the wet season accumulated rainfall. The explained variance was calculated, using lineal multiple regression and cross-validation (Montgomery, 2001). Results suggest that correlated anomalies are associated with the Intertropical Convergence Zone movement. A 26-year precipitation time series at Huayao weather station, Chupaca province, was used. TNA (Tropical North Atlantic) and TSA (Tropical South Atlantic) indices were used as sea surface temperature anomalies indicators, as described by NOAA's ESRL (Earth Science Research Laboratory).
\end{abstract}

Keywords: Mantaro Valley rainfall; oceanic indices; TSA Tropical South Atlantic index; TNA tropical North Atlantic index.

\section{Introducción}

La precipitación en el valle del Mantaro tiene gran importancia por su efecto en la agricultura, ya que esta es la fuente de muchos productos que son consumidos en Lima. En diferentes estudios se ha tratado de relacionar la precipitación con eventos diversos, uno de ellos El Niño (Lavado, 2010), pero sin encontrar un efecto significativo. Es conocido que las precipitaciones en el valle que estudiamos provienen de masas de aire que llegan de la Amazonía y, probablemente, del océano Atlántico (Lavado et al., 2011) pudiendo haber relación entre un evento extremo en el Atlántico y la sequía en el río Amazonas en el 2010. En consecuencia, el objetivo de este trabajo es el de encontrar una relación entre la precipitación y las anomalías mensuales de la temperatura superficial del océano Atlántico tropical, tal como están definidas por la división de ciencias físicas del laboratorio de investigación de ciencias de la tierra (ESRL) de la NOAA.

\section{Materiales y métodos}

Se ha usado información de precipitación mensual de la estación de Huayao en la provincia de Chupaca, región Junín, debido a que es la estación más representativa del valle, tal como lo demuestran los múltiples estudios realizados en la zona. La serie de datos abarca el periodo que va desde 1983/1984 hasta el 2008/2009. Además, se ha sumado la precipitación de los seis meses más lluviosos para tener la precipitación total de la temporada húmeda (octubre-marzo). La información del océano atlántico está dada por los índices mensuales TSA y TNA (Atlántico

1 Profesor del Departamento de Ingeniería Ambiental Física y Meteorología. Universidad Nacional Agraria La Molina, Lima, Perú. Email: jcchang@lamolina.edu.pe. 
Sur tropical y Atlántico Norte tropical), los cuales están basados en la anomalía de temperatura de la superficie del mar en las siguientes regiones: Atlántico tropical Sur, 0-20S, 13W-57,5W; Atlántico tropical Norte, 5,5N-23,5N y $15 \mathrm{~W}-57,5 \mathrm{~W}$ (ESRL, 2012). Para la regresión múltiple y sus variantes se usó Minitab 15. El software usado para la validación cruzada fue la herramienta de predictibilidad climática (Climate Predictability Tool) del International Research Institute (IRI) de la Universidad de Columbia. El software por "default" utiliza 500 submuestras para la validación cruzada. El criterio inicial para la selección de variables predictoras o explicativas fue el de un error estándar (S) bajo. Posteriormente, se comprobaron los valores $\mathrm{p}$ de las pruebas $\mathrm{F}$ de los diferentes modelos de regresión hallados. Para evitar valores excesivamente altos del coeficiente de determinación o variancia explicada, se aplicó una validación cruzada usando submuestras cuyo número de observaciones fue similar al de los grados de libertad del error residual.

\section{Resultados y discusión}

La ec. 1 presenta la ecuación hallada y la Tabla 1 muestra las variables escogidas para el modelo. En la Tabla 2 encontramos los estadísticos de los coeficientes de regresión; mientras que la Tabla 3 presenta los estadísticos del modelo en su conjunto: error estándar, coeficiente de determinación, coeficiente de determinación ajustado, coeficiente de determinación reducido por validación cruzada.

$p p=540,54-379,37 t s a 09+540,30 t s a 10-302,2 t s a 12+$ 224,98 tsa01 -132,33tn02 (ec.1)

Tabla 1. Variables incorporadas al modelo escogido

\begin{tabular}{ll}
\hline Nombre & Significado \\
$\mathrm{pp}$ & Precipitación acumulada octubre-marzo \\
tsa09 & Índice del Atlántico tropical Sur de setiembre \\
tsa10 & Índice del Atlántico tropical Sur de octubre \\
tsa12 & Índice del Atlántico tropical Sur de diciembre \\
tsa01 & Índice del Atlántico tropical Sur de enero \\
tna02 & Índice del Atlántico tropical Norte de febrero \\
\hline
\end{tabular}

Tabla 2. Estadísticos de los coeficientes de regresión

\begin{tabular}{lrrr}
\hline Predictor & Coeficiente & valor t & $\mathrm{p}$ \\
Intercepto & 540,54 & 37,09 & 0,000 \\
tsa09 & $-379,37$ & $-4,94$ & 0,000 \\
tsa10 & 540,30 & 5,49 & 0,000 \\
tsa12 & $-302,2$ & $-2,98$ & 0,007 \\
tsa01 & 224,98 & 2,92 & 0,008 \\
tna02 & $-133,33$ & $-4,14$ & 0,001 \\
\hline
\end{tabular}

Tabla 3. Estadísticos globales del modelo

\begin{tabular}{|l|r|}
\hline Error estándar S & 55,8 \\
\hline $\mathrm{R}^{2}$ & 0,755 \\
\hline $\mathrm{R}^{2}$ ajustado & 0,694 \\
\hline $\mathrm{R}^{2}$ validación cruzada & 0,647 \\
\hline
\end{tabular}

La Tabla 4 presenta el análisis de variancia del modelo de regresión. Con una confianza del $95 \%$, el valor inferior de la variancia explicada es de $40 \%$ y el valor superior de $80 \%$.

Tabla 4. Análisis de variancia de la regresión

\begin{tabular}{lrrrrr}
\hline Fuente & GL & SC & CM & F & p \\
\hline Regresión & 5 & 192475 & 38495 & 12,34 & 0,00015 \\
Error residual & 20 & 62374 & 3119 & & \\
Total & 25 & 254849 & & & \\
\hline
\end{tabular}

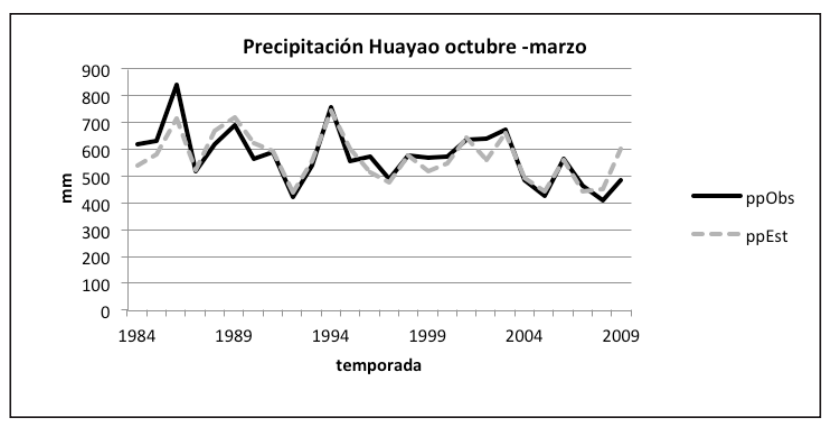

Figura 1. Valores observados y estimados por el modelo de regresión múltiple

Además de la fuerte variancia explicada, resalta que de las cinco variables causales o "predictoras", las primeras cuatro pertenecen al océano Atlántico tropical Sur. Las anomalías del Atlántico tropical Norte son importantes en febrero. Probablemente, se deba a la existencia de un gradiente térmico entre las zonas halladas en el modelo y la zona de convergencia intertropical en los meses de enero a marzo. También es importante destacar que el índice tsa de setiembre tenga un efecto en la precipitación de la estación lluviosa, indicando cierto retardo entre la ocurrencia de la anomalía y la ocurrencia de la precipitación. Respecto a los coeficientes de regresión, todos presentan un valor $p$ inferior a 0,01 , lo cual significa que el modelo es robusto (Montgomery, 2001). Lo que resulta difícil de explicar son los signos de estos coeficientes; en algunos casos la relación es directa (octubre, enero) $\mathrm{y}$, en otros, inversa (setiembre, diciembre, febrero). Es posible que el signo negativo de los coeficientes para los meses de octubre y enero sea su colinearidad con las variables de setiembre y febrero, por lo que el análisis de regresión les ha asignado signos contrarios. 
Tabla 5. Regresión de los mejores subconjuntos

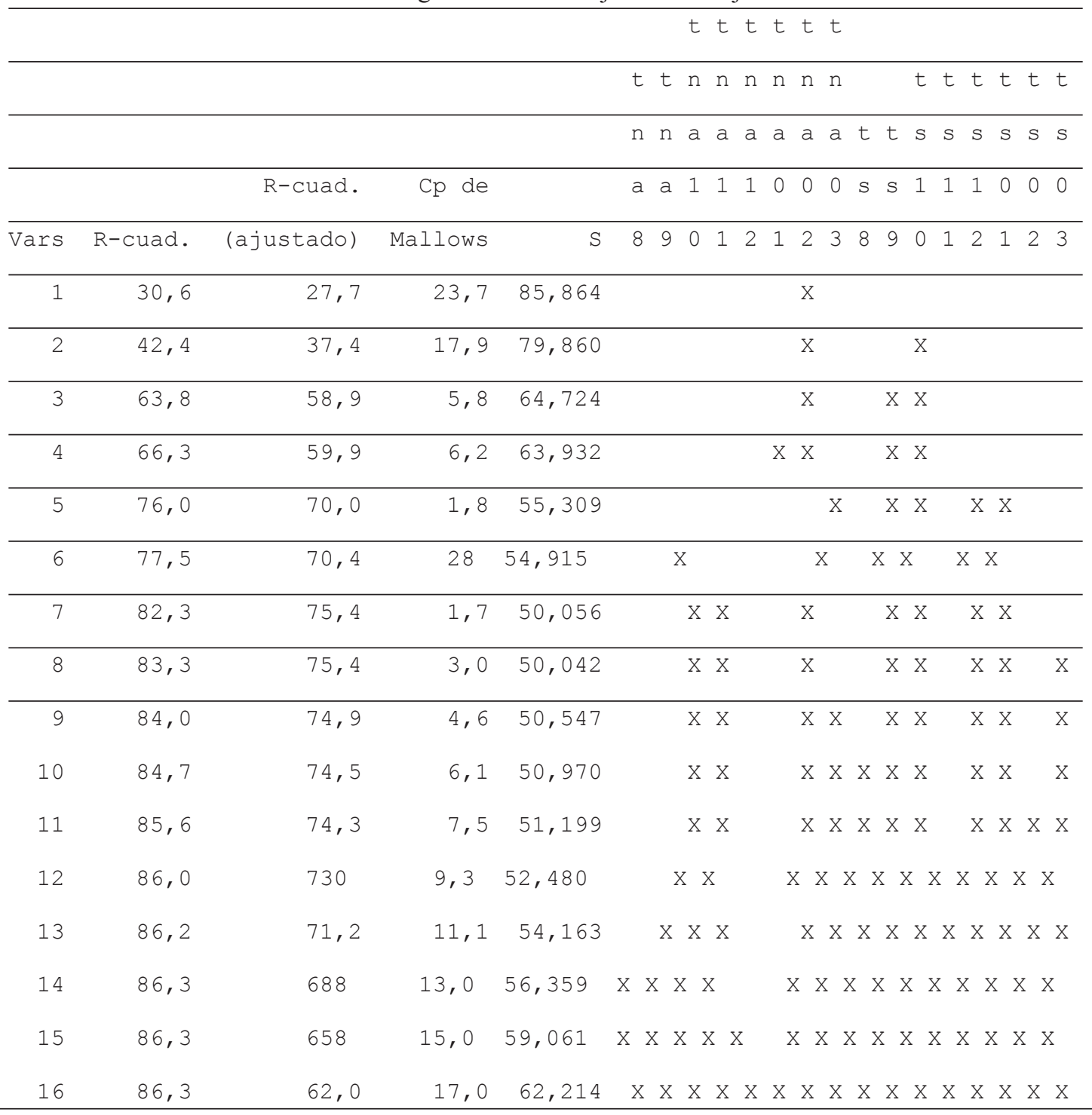

La Tabla 5 presenta los estadísticos de los mejores modelos con diferentes números de variables. Al observar los modelos con no más de seis variables, se desprende que las anomalías del Atlántico Norte (tnaXX) tienen más incidencia en los meses de enero a marzo, mientras que los del Sur (tsaXX) tienen más incidencia en los meses de setiembre y octubre.

\section{Conclusiones}

La variabilidad de las anomalías de temperatura superficial del océano Atlántico tropical, en especial del hemisferio sur, explica en promedio, el $64,7 \%$ de la variabilidad de la precipitación en el valle del Mantaro. Respecto a las variables predictoras, hay una preponderancia de las anomalías del Atlántico tropical Sur, a inicios de la campaña húmeda, mientras que a finales de esta la preponderancia es de las anomalías del Atlántico tropical Norte, lo cual indicaría que las anomalías de temperaturas están relacionadas con la región tropical complementaria a la zona de convergencia intertropical.

\section{Literatura citada}

National Oceanic \& Atmospheric Administration [NOAA]. 2012. Laboratorio de Investigación de Ciencias de la Tierra (ESRL). (2012). Climate Indices. Recuperado de http://www.esrl.noaa.gov/psd/data/climateindices/list/

Espinoza, J. et al. 2011. Las recientes sequías en la cuenca amazónica peruana: Orígenes climáticos e impactos hidrológicos. Revista Peruana Geo-Atmosférica, $\mathbf{N}^{\circ} 3$. Lima, Perú: SENAMHI.

Lavado, W. et al. 2010. Impacto de ENOS en la Hidrología del Perú (1968-2006). Revista Peruana Geo-Atmosférica, No 1. Lima, Perú: SENAMHI.

Montgomery, D. et al. 2001. Introducción al Análisis de Regresión Lineal. México: Grupo Editorial Patria. 